\title{
Does the use of preoperative bisphosphonates in patients with osteopenia and osteoporosis affect lumbar fusion rates? Analysis from a national spine registry
}

\author{
Kern H. Guppy, MD, PhD, ${ }^{1}$ Priscilla H. Chan, MS, ${ }^{2}$ Heather A. Prentice, PhD, ${ }^{2}$ \\ Elizabeth P. Norheim, MD, ${ }^{3}$ Jessica E. Harris, MS, RD, ${ }^{2}$ and Harsimran S. Brara, MD ${ }^{4}$ \\ 'Department of Neurosurgery, The Permanente Medical Group, Sacramento; ${ }^{2}$ Surgical Outcomes \& Analysis, Kaiser \\ Permanente, San Diego; ${ }^{3}$ Department of Spinal Surgery, Southern California Permanente Medical Group, Downey; and \\ ${ }^{4}$ Department of Neurosurgery, Southern California Permanente Medical Group, Los Angeles, California
}

\begin{abstract}
OBJECTIVE Bisphosphonates are used to increase bone strength in treating osteopenia and osteoporosis, but their use for increasing lumbar fusion rates has been controversial. The objective of this study was to determine if preoperative treatment with bisphosphonates affects the reoperation rates for nonunions (operative nonunion rates) following lumbar fusions in patients with osteopenia or osteoporosis.

METHODS The authors conducted a cohort study using data from the Kaiser Permanente Spine Registry. Patients (aged $\geq 50$ years) with a diagnosis of osteopenia or osteoporosis who underwent primary elective lumbar fusions for degenerative disc disease, deformity, or spondylolisthesis were included in the cohort. Repeated spinal procedures at the index lumbar levels were noted through chart review. Reoperations for symptomatic nonunions (operative nonunions), time to nonunion, and the nonunion spine level(s) were also identified. The crude 2-year cumulative incidence of operative nonunions was calculated as 1 minus the Kaplan-Meier estimator. Cox proportional hazard regression was used to evaluate the association between preoperative bisphosphonate use and operative nonunion after adjustment for covariates. Analysis was stratified by osteopenia and osteoporosis diagnosis.
\end{abstract}

RESULTS The cohort comprised 1040 primary elective lumbar fusion patients, 408 with osteopenia and 632 with osteoporosis. Ninety-seven (23.8\%) patients with osteopenia and $370(58.5 \%)$ patients with osteoporosis were preoperative bisphosphonate users. For the osteopenia group, no operative nonunions were observed in patients with preoperative bisphosphonate, while the crude 2-year incidence was $2.44 \%(95 \% \mathrm{Cl} 0.63-4.22)$ in the nonuser group. For the osteoporotic group, after adjustment for covariates, no difference was observed in risk for operative nonunions between the preoperative bisphosphonate users and nonusers ( $\mathrm{HR} 0.96,95 \% \mathrm{Cl} 0.20-4.55, \mathrm{p}=0.964$ ).

CONCLUSIONS To the authors' knowledge, this study presents one of the largest series of patients with the diagnosis of osteopenia or osteoporosis in whom the effects of preoperative bisphosphonates on lumbar fusions were evaluated using operative nonunion rates as an outcome measure. The results indicate that preoperative bisphosphonate use had no effect on the operative nonunion rates for patients with osteoporosis. Similar indications were not confirmed in osteopenia patients because of the low nonunion frequency. Further studies are warranted to the determine if preoperative and postoperative timing of bisphosphonate use has any effect on lumbar fusion rates.

https://thejns.org/doi/abs/10.3171/2020.5.FOCUS20262

KEYWORDS bisphosphonates; osteopenia; osteoporosis; reoperation; pseudarthrosis; nonunion; spine registry; retrospective; lumbar fusion

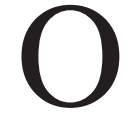
STEOPENIA and osteoporosis are increasingly common comorbidities in patients undergoing elective spine fusion surgery, due to the demographic shift to an older population in the United States. "Osteopenia" and "osteoporosis" are terms used to describe decreased bone density determined by measuring the amount of calcium in bones to estimate the risk of bone fractures. The most widely used test for estimating bone density is dualenergy x-ray absorptiometry (DEXA), which reports a T score. $\mathrm{T}$ scores greater than -1 indicate normal bone den-

ABBREVIATIONS ALIF = anterior lumbar interbody fusion with pedicle screws; $\mathrm{BMP}=$ bone morphogenic protein; $\mathrm{CIF}=$ cumulative incidence function; $\mathrm{CPT}=\mathrm{Current}$ Procedural Terminology; DEXA = dual-energy x-ray absorptiometry; EHR = electronic health record; PLF = posterolateral lumbar fusion with pedicle screws; PLIF = posterior lumbar interbody fusion with pedicle screws; RCT = randomized controlled trial; TLIF = transforaminal lumbar interbody fusion with pedicle screws; ZA = zoledronic acid. ACCOMPANYING EDITORIAL DOI: 10.3171/2020.5.FOCUS20413.

SUBMITTED April 2, 2020. ACCEPTED May 12, 2020.

INCLUDE WHEN CITING DOI: 10.3171/2020.5.FOCUS20262. 
sity, scores between -1 and -2.49 indicate osteopenia, and scores less than or equal to -2.5 indicate osteoporosis. The incidence of osteoporosis in adults aged $>50$ years has been reported to be $14.5 \%$ in men and $51.3 \%$ in women. ${ }^{1}$

Complications from lumbar fusions in patients with osteopenia and osteoporosis are well documented. Vertebral compression fractures, cage subsidence, and loosening of pedicle screws are frequently reported after lumbar fusions. ${ }^{2-4}$ These complications have been attributed to low bone density, and recently spinal implants and medications have been developed to address this problem. Bisphosphonates have being widely prescribed and are often considered the first line of treatment for osteopenia and osteoporosis. ${ }^{5}$ Bisphosphonates work by preventing bone resorption and remodeling, stopping osteoclast activity, and disrupting the balance between osteoclastic and osteoblastic activities. ${ }^{6,7}$ The appropriate use of this class of medications in the perioperative period is the subject of intense study. It would be expected that bisphosphonates that prevent bone loss would lead to better fusions; however, there have been conflicting findings in both animal and clinical studies, with no consensus on the influence of bisphosphonates on successful spinal arthrodesis. ${ }^{8-25}$

Many of these clinical investigations use radiographic studies such as plain flexion-extension radiographs and CT scans to determine fusion rates. However, the correlations between radiographic nonunions and clinical outcomes have also been debated. The determination of true nonunion rates requires surgical exploration with direct visualization of bone fusions. ${ }^{26,27}$ Dawson et al. ${ }^{28}$ noted that the radiographic definition of "nonunion" may have been too stringent in many of the studies reported in the literature and may not be an accurate representation of normal clinical practices. Singh et al. ${ }^{29}$ also noted that there may be inherent biases in decision-making with regard to identifying nonunion in both investigational and control groups of randomized controlled trials (RCTs) that may be different from general surgical practices. In many cases, patients with radiographic nonunion are asymptomatic and, therefore, a more clinically useful measure would be combining clinical and radiographic outcomes by using reoperation rates for symptomatic nonunion (operative nonunion). ${ }^{30-33}$

Therefore, in our study we identified a cohort of patients with a diagnosis of either osteopenia or osteoporosis who underwent primary elective lumbar fusions and evaluated the association between preoperative bisphosphonate use and operative nonunions.

\section{Methods}

We conducted a retrospective cohort study using data from the Kaiser Permanente Spine Registry. Kaiser Permanente is an integrated healthcare system that covers 12.2 million members throughout 8 geographical regions (Colorado, Georgia, Hawaii, the Mid-Atlantic, Northern California, the Northwest, Southern California, and Washington) in the United States. This patient population has been shown to be socioeconomically and demographically representative for the geographic regions it covers. $^{34,35}$

\section{Data Source}

The data collection and validation processes have been previously described. ${ }^{36}$ Briefly, predefined detailed patient- and procedure-related information for instrumented spine procedures performed within our institutions are collected at the point of care by the operating surgeon via electronic intraoperative forms. Implant data are captured into the registry from the implant module of the electronic health records (EHRs). Additional data are then supplemented using information from the EHRs, administrative claims data, membership data, and mortality records.

The pharmacy module of Kaiser Permanente's integrated EHR was the second data source for the study. This EPIC-based platform captures data for all medications prescribed and dispensed within the healthcare system.

\section{Inclusion and Exclusion Criteria}

All patients aged $\geq 50$ years with a diagnosis of either osteopenia or osteoporosis who underwent primary elective single-level or multilevel lumbar fusions for the diagnosis of deformity, spondylolisthesis, or stenosis from 2009 to 2016 comprised the study cohort. Procedures included posterolateral lumbar fusions with pedicle screws (PLFs), posterior lumbar interbody fusions with pedicle screws (PLIFs), transforaminal lumbar interbody fusions with pedicle screws (TLIFs), and anterior lumbar interbody fusions with pedicle screws (ALIFs). Patients with spinal tumors, infection, or trauma, as well as those with fusions performed in conjunction with the cervical or thoracic spine, stand-alone ALIF, combined TLIFs and PLIFs, nonfusion procedures (decompression only), or staged procedures, were all excluded. For simplicity and to focus only on lumbar fusions with pedicle screws (Tables 1 and 2), we categorized our fusions into PLF, TLIF or PLIF (either PLIF or TLIF, not a combination of both), and ALIF (not stand-alone ALIF).

Bone quality (osteopenia or osteoporosis) was obtained using the following Current Procedural Terminology (CPT) and/or ICD-10 clinical modification codes: osteopenia (CPT code 733.90 or ICD-10 code M85.8) and osteoporosis (CPT codes 733.00 and 733.01 [age dependent] or ICD-10 code M81.0). Patients with no diagnosis (no CPT or ICD code) were excluded. If a patient had any of the specified codes documented in our healthcare system's integrated EHR prior to the date of the index procedure, then they were considered as having osteopenia or osteoporosis. Codes could have been entered into the patient's health record at any prior healthcare encounter within the health system and were not necessarily specific to just the spine procedure.

\section{Exposure of Interest}

Preoperative bisphosphonate use, as determined though medication prescription and date dispensed, was the exposure of interest. Bisphosphonates included alendronate (with or without cholecalciferol), etidronate, ibandronate, pamidronate, risedronate (with or without calcium carbonate), and zoledronic acid (ZA; with or without mannitol and water). All other medications for the treatment 
TABLE 1. Demographics of osteopenic lumbar fusion patients with and without preoperative bisphosphonates

\begin{tabular}{|c|c|c|c|}
\hline \multirow[b]{2}{*}{ Demographic Data } & \multirow[b]{2}{*}{ All Patients } & \multicolumn{2}{|c|}{ Preoperative Bisphosphonates } \\
\hline & & No & Yes \\
\hline No. of patients & 408 & 311 & 97 \\
\hline \multicolumn{4}{|l|}{ Age, yrs } \\
\hline Mean (SD) & $68.9(7.6)$ & $68.6(7.5)$ & $69.9(8.1)$ \\
\hline Median (IQR) & $69(64-75)$ & $69(64-74)$ & $72(64-75)$ \\
\hline Female sex & $323(79.2 \%)$ & $237(76.2 \%)$ & $86(88.7 \%)$ \\
\hline \multicolumn{4}{|l|}{$\mathrm{BMI}, \mathrm{kg} / \mathrm{m}^{2}$} \\
\hline Mean (SD) & $27.2(4.9)$ & $27.5(5.0)$ & $26.0(4.3)$ \\
\hline Median (IQR) & $26.6(23.8-29.8)$ & $27.0(24.3-30.5)$ & $25.5(23.0-27.9)$ \\
\hline \multicolumn{4}{|l|}{ Admitting diagnosis* } \\
\hline Adult deformity & $75(18.4 \%)$ & $53(17.0 \%)$ & $22(22.7 \%)$ \\
\hline Spondylolisthesis & $205(50.2 \%)$ & $166(53.4 \%)$ & $39(40.2 \%)$ \\
\hline Stenosis & $307(75.2 \%)$ & $237(76.2 \%)$ & $70(72.7 \%)$ \\
\hline \multicolumn{4}{|l|}{ Operative time, mins $†$} \\
\hline$<100$ & $10(2.5 \%)$ & $10(3.3 \%)$ & $0(0.0 \%)$ \\
\hline $100-199$ & $183(45.5 \%)$ & $135(44.1 \%)$ & $48(50.0 \%)$ \\
\hline $200-299$ & $144(35.8 \%)$ & $107(35.0 \%)$ & $37(38.5 \%)$ \\
\hline$\geq 300$ & $65(16.2 \%)$ & $54(17.6 \%)$ & $11(11.5 \%)$ \\
\hline \multicolumn{4}{|l|}{ No. of levels fused } \\
\hline 1 & $215(52.7 \%)$ & $165(53.1 \%)$ & $50(51.5 \%)$ \\
\hline 2 & $112(27.5 \%)$ & $92(29.6 \%)$ & $20(20.6 \%)$ \\
\hline 3 & $45(11.0 \%)$ & $30(9.6 \%)$ & $15(15.5 \%)$ \\
\hline 4 & $24(5.9 \%)$ & $16(5.1 \%)$ & $8(8.2 \%)$ \\
\hline 5 & $12(2.9 \%)$ & $8(2.6 \%)$ & $4(4.1 \%)$ \\
\hline \multicolumn{4}{|l|}{ Surgical approach } \\
\hline PLF & $172(42.2 \%)$ & $129(41.5 \%)$ & $43(44.3 \%)$ \\
\hline ALIF $\ddagger$ & $48(11.8 \%)$ & $34(10.9 \%)$ & $14(14.4 \%)$ \\
\hline PLIF or TLIF & $188(46.1 \%)$ & $148(47.6 \%)$ & $40(41.2 \%)$ \\
\hline BMP use & $304(74.5 \%)$ & $228(73.3 \%)$ & $76(78.4 \%)$ \\
\hline Smoker & $18(4.4 \%)$ & $16(5.1 \%)$ & $2(2.1 \%)$ \\
\hline
\end{tabular}

Values are presented as number of patients (\%) unless otherwise indicated.

* Diagnoses based on ICD-10 codes. Patients could have > 1 diagnosis.

$\dagger$ Missing operative time data for $6(1.5 \%)$ patients.

$\ddagger$ ALIF with pedicle screws.

of osteoporosis, such as anabolic agents like teriparatide, were excluded. Patients were defined as preoperative bisphosphonate users if they had at least a 6-month bisphosphonate supply and at least $80 \%$ adherence during that time (proportion of days between first and last prescription) by the time of index surgery. Nonusers included patients who were never offered bisphosphonates, those who may have been offered bisphosphonates but refused (no medication dispensed), those who had poor adherence and compliance, those on a drug holiday as prescribed by their physician, or those who could not tolerate the medication (adherence less than $80 \%$ of the time). Bisphosphonate intolerance occurs frequently, with patients having adverse symptoms such as gastrointestinal intolerance, atrial fibrillation, transient increase in serum creatine levels, or incidents of atypical femur fractures. ${ }^{37-39}$

\section{Outcome of Interest}

The primary outcome of interest was reoperation for nonunions (operative nonunions). In the registry, the reasons for all reoperations (nonunions, adjacent segment disease, etc.) are recorded by the operating surgeon using intraoperative forms at the time of surgery. For this study, charts were adjudicated via manual EHR review by a trained clinical research associate to ensure accuracy, and only reoperations for nonunions were recorded. Following their index lumbar fusion, all patients were continuously monitored for reoperations until death, loss to follow-up through healthcare membership termination, or study end date (March 31, 2018).

\section{Covariates}

Patient and surgical covariates considered potential 
TABLE 2. Demographic characteristics of osteoporotic lumbar fusion patients with and without preoperative bisphosphonates

\begin{tabular}{|c|c|c|c|}
\hline \multirow[b]{2}{*}{ Demographic Data } & \multirow[b]{2}{*}{ All Patients } & \multicolumn{2}{|c|}{ Preoperative Bisphosphonates } \\
\hline & & No & Yes \\
\hline No. of patients & 632 & 262 & 370 \\
\hline \multicolumn{4}{|l|}{ Age, yrs } \\
\hline Mean (SD) & $71.5(7.9)$ & $70.6(8.5)$ & $72.1(7.5)$ \\
\hline Median (IQR) & $72(66-77)$ & $70.5(65-77)$ & $72(67-77)$ \\
\hline Female sex & $535(84.7 \%)$ & $206(78.6 \%)$ & $329(88.9 \%)$ \\
\hline \multicolumn{4}{|l|}{$\mathrm{BMI}, \mathrm{kg} / \mathrm{m}^{2}$} \\
\hline Mean (SD) & $26.2(4.8)$ & $27.1(4.9)$ & $25.6(4.7)$ \\
\hline Median (IQR) & $25.4(22.7-29.0)$ & $26.4(23.6-29.6)$ & $24.8(22.1-28.4)$ \\
\hline \multicolumn{4}{|l|}{ Admitting diagnosis* } \\
\hline Adult deformity & $150(23.7 \%)$ & $57(21.8 \%)$ & $93(25.1 \%)$ \\
\hline Spondylolisthesis & $290(45.9 \%)$ & $120(45.8 \%)$ & $170(45.9 \%)$ \\
\hline Stenosis & $443(70.1 \%)$ & $183(69.8 \%)$ & $260(70.3 \%)$ \\
\hline \multicolumn{4}{|l|}{ Operative time, mins $\dagger$} \\
\hline$<100$ & $21(3.5 \%)$ & $5(2.0 \%)$ & $16(4.5 \%)$ \\
\hline $100-199$ & $299(49.6 \%)$ & $122(49.4 \%)$ & $177(49.7 \%)$ \\
\hline $200-299$ & $209(34.7 \%)$ & $82(33.2 \%)$ & $127(35.7 \%)$ \\
\hline$\geq 300$ & $74(12.3 \%)$ & $38(15.4 \%)$ & $36(10.1 \%)$ \\
\hline \multicolumn{4}{|l|}{ No. of levels fused } \\
\hline 1 & $341(54.0 \%)$ & $138(52.7 \%)$ & $203(54.9 \%)$ \\
\hline 2 & $179(28.3 \%)$ & $71(27.1 \%)$ & $108(29.2 \%)$ \\
\hline 3 & $62(9.8 \%)$ & $29(11.1 \%)$ & $33(8.9 \%)$ \\
\hline 4 & $44(7.0 \%)$ & $21(8.0 \%)$ & $23(6.2 \%)$ \\
\hline 5 & $6(0.9 \%)$ & $3(1.1 \%)$ & $3(0.8 \%)$ \\
\hline \multicolumn{4}{|l|}{ Surgical approach } \\
\hline PLF & $294(46.5 \%)$ & $124(47.3 \%)$ & $170(45.9 \%)$ \\
\hline ALIF $\ddagger$ & $66(10.4 \%)$ & $34(13.0 \%)$ & $32(8.6 \%)$ \\
\hline PLIF or TLIF & $272(43.0 \%)$ & $104(39.7 \%)$ & $168(45.4 \%)$ \\
\hline BMP use & $467(73.9 \%)$ & $195(74.4 \%)$ & $272(73.5 \%)$ \\
\hline Smoker & $25(4.0 \%)$ & $12(4.6 \%)$ & $13(3.5 \%)$ \\
\hline
\end{tabular}

Values are presented as number of patients (\%) unless otherwise indicated.

* Diagnoses based on ICD-10 codes. Patients could have > 1 diagnosis.

$\dagger$ Missing operative time data for $29(4.6 \%)$ patients.

$\ddagger$ ALIF with pedicle screws.

confounding factors included age at index surgery (continuous), BMI (in $\mathrm{kg} / \mathrm{m}^{2}$; continuous), sex (male/female), tobacco smoking history, admitting diagnosis (adult deformity, spondylolisthesis, or stenosis), operating time in minutes (<100, 100-199, 200-299, or $\geq 300)$, surgical approach (PLF, ALIF, or PLIF or TLIF), use of bone morphogenic protein (BMP), and number of levels fused.

\section{Statistical Analysis}

The cumulative incidence function (CIF) for the crude 2 -year operative nonunion rates was calculated as $1 \mathrm{mi}$ nus the Kaplan-Meier estimator. The risk for operative nonunion at any point during follow-up was modeled as a time-to-event outcome using Cox proportional hazards regression. Hazard ratios (HRs) and 95\% confidence intervals (CIs) are presented; analysis was stratified by os- teopenia and osteoporosis diagnosis. Regression models included the exposure of interest and the potential confounders listed above. Follow-up time was defined as the time from the date of the index surgery to the operative nonunion date, healthcare membership termination date, death date, or study end date, whichever came first. Analyses were performed using $\mathrm{R}$ version 3.5.2. All tests were 2 -sided and statistical significance was defined as $\mathrm{p}<0.05$.

\section{Results}

The cohort comprised 1040 primary elective lumbar fusion patients, 408 with osteopenia and 632 with osteoporosis. Fusions included in the study sample were performed by 93 surgeons at 17 healthcare centers. Ninetyseven $(23.8 \%)$ patients with osteopenia and $370(58.5 \%)$ 


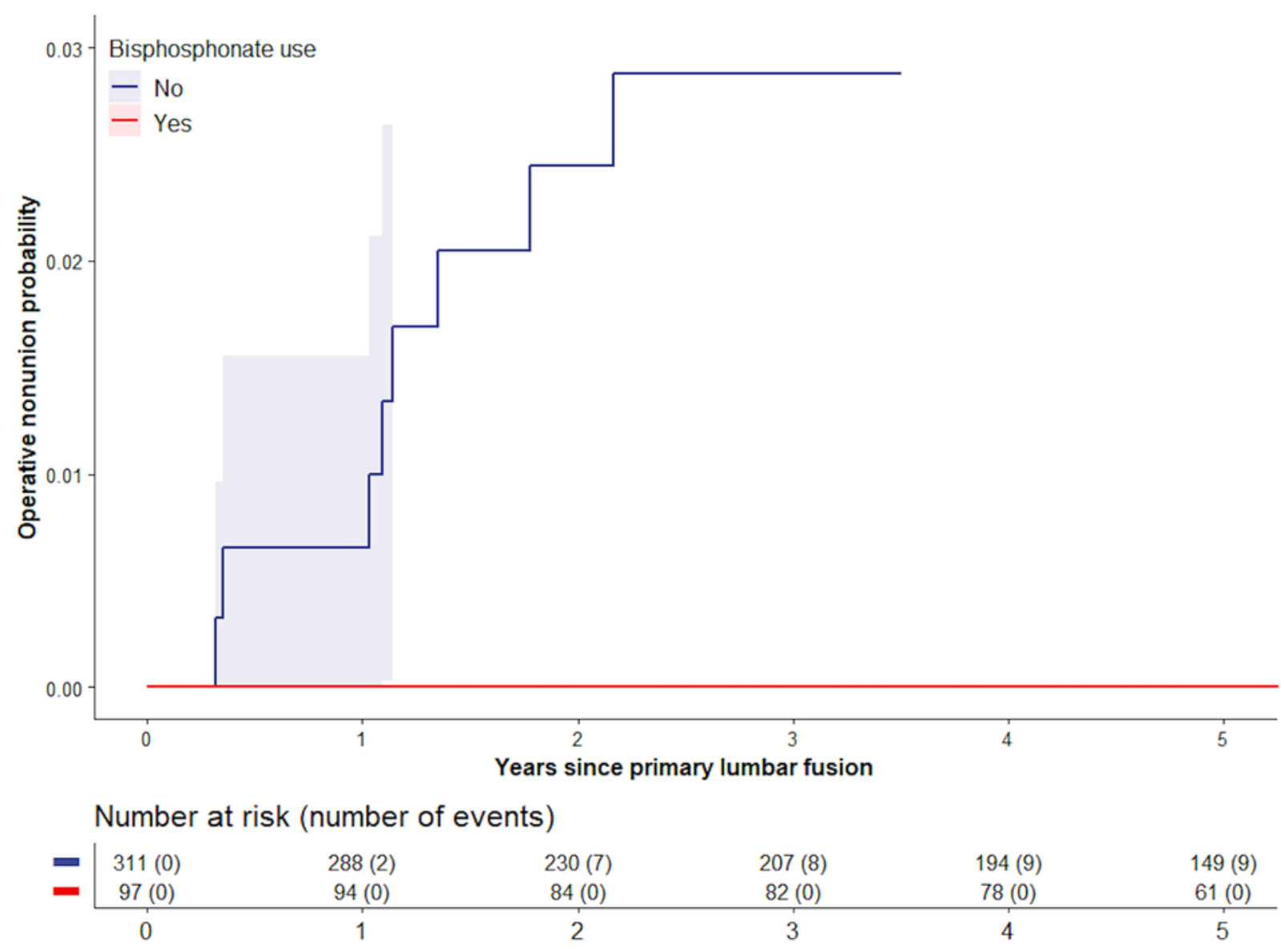

FIG. 1. Kaplan-Meier graph showing the incidence of operative nonunions during follow-up for patients with osteopenia.

patients with osteoporosis were preoperative bisphosphonate users.

For patients with osteopenia, the mean (SD) age and BMI were 68.9 (7.6) years and $27.2(4.9) \mathrm{kg} / \mathrm{m}^{2}$, respectively. The majority of patients with osteopenia were female (79.2\%), nonsmokers (95.6\%), and had a diagnosis of spondylolisthesis $(50.2 \%)$ or stenosis $(75.2 \%)$. The most frequently performed procedures were PLIFs/TLIFs $(46.1 \%)$ and single-level fusions (52.7\%). BMP use was similar between bisphosphonate users and nonusers (Table 1).

The mean (SD) age and BMI for patients with osteoporosis were 71.5 (7.9) years and $26.2(4.8) \mathrm{kg} / \mathrm{m}^{2}$, respectively. The majority of patients with osteoporosis were female (84.7\%), nonsmokers $(96.0 \%)$, and had a diagnosis of stenosis $(70.1 \%)$. More patients underwent PLFs $(46.5 \%)$ and had a single-level fusion (54.0\%). BMP use was similar in both groups (Table 2).

The median follow-up for the entire cohort was 5.2 years, with a maximum follow-up of 9.2 years. Figures 1 and 2 present the incidence of operative nonunions during follow-up for patients with osteopenia and osteoporosis, respectively. No operative nonunions were observed following lumbar fusion in patients with osteopenia who used bisphosphonates; the crude 2-year incidence was $2.44 \%$ (95\% CI 0.63-4.22) for nonusers. The crude 2-year incidence of operative nonunions for patients with osteoporosis was $0.85 \%$ (95\% CI $0.00-1.80$ ) for bisphosphonate users and $1.89 \%$ (95\% CI $0.03-3.71$ ) for nonusers. The Cox regression model was adjusted for age, BMI, sex, admitting diagnosis, operative time, surgical approach, BMP use, and number of levels fused (Table 3). No difference was observed in risk for operative nonunion when preoperative bisphosphonate users were compared with nonusers with osteoporosis (HR $0.96,95 \%$ CI $0.20-4.55$, $\mathrm{p}=0.964$ ). Adjusted analysis for patients with osteopenia could not be performed due to there being no events in the bisphosphonate user group.

\section{Discussion}

In our cohort of patients with osteopenia who had preoperative bisphosphonates, we did not find any operative nonunions; however, in patients without preoperative bisphosphonates, the crude 2-year incidence of operative nonunions was $2.44 \%$ (Table 3). Although there is a trend for preoperative bisphosphonate to be protective in osteopenia patients, we were unable to come to a conclusion because no adjusted analysis could be made on the risks of operative nonunions. Our study is unique in that it is, to our knowledge, the first study that has looked at the effects of preoperative bisphosphonates in patients with 


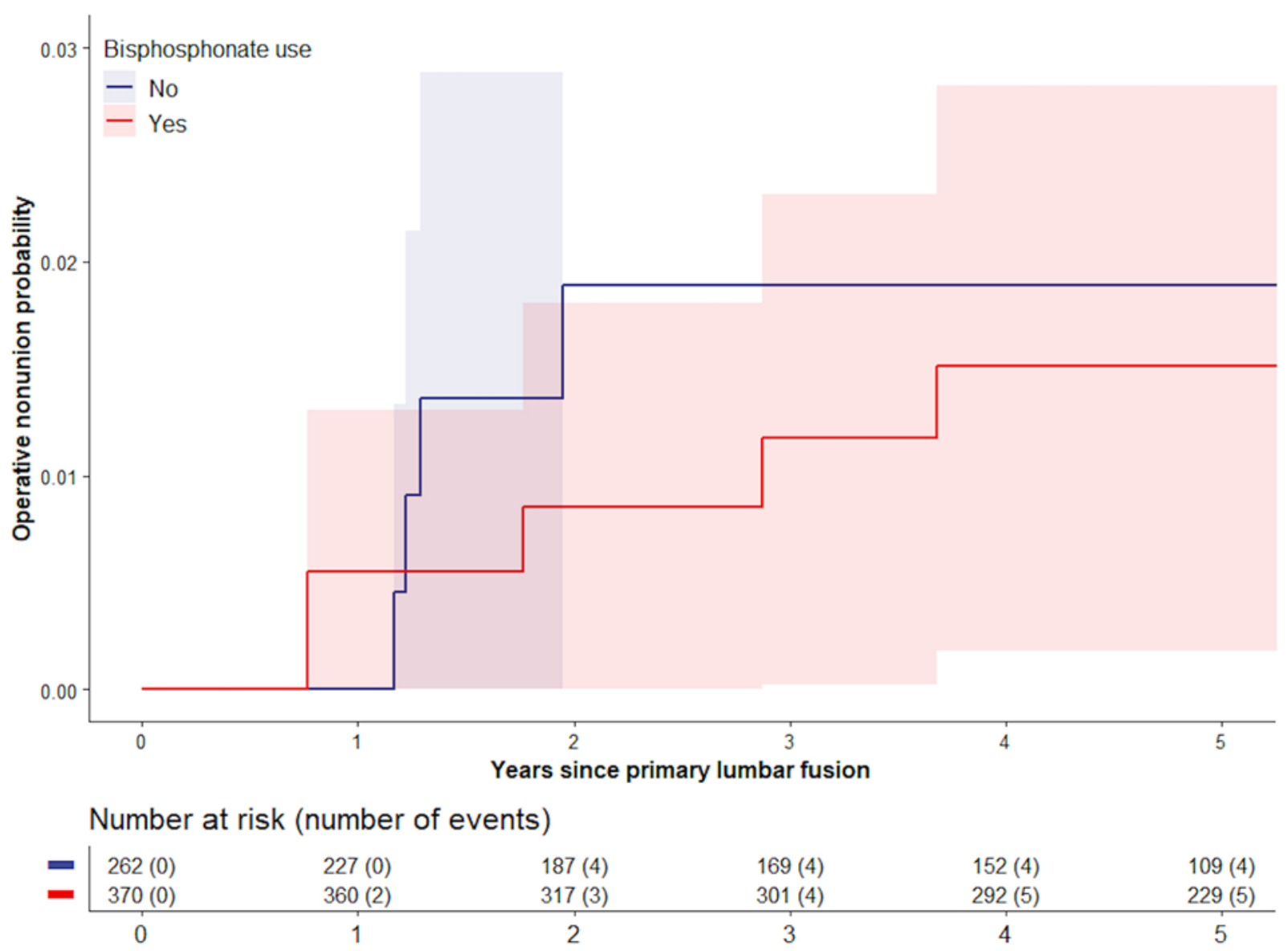

FIG. 2. Kaplan-Meier graph showing the incidence of operative nonunions during follow-up for patients with osteoporosis.

osteopenia. This is not surprising since the guidelines for bisphosphonate use in osteopenic patients have not been universally accepted, although there have been studies that have shown its efficacy in reducing nonvertebral and vertebral fractures. ${ }^{40,41}$ The National Osteoporosis Foun- dation guidelines of 2014 (https://cdn.nof.org/wp-content/ uploads/2016/01/995.pdf) did endorse pharmaceutical intervention in women with osteopenia, although the guidelines noted that in this group of patients there were relatively few data confirming that fracture risk reductions

TABLE 3. Crude nonunion incidence rates and adjusted association between preoperative bisphosphonate use and adjusted risks of operative nonunions

\begin{tabular}{lccccc}
\hline & $\begin{array}{c}\text { No. of } \\
\text { Fusions }\end{array}$ & $\begin{array}{c}\text { No. of } \\
\text { Nonunions }\end{array}$ & $\begin{array}{c}2-Y r \text { Nonunion ClF* } \\
(95 \% \mathrm{Cl})\end{array}$ & HR† $(95 \% \mathrm{Cl})$ & p Value \\
\hline Total no. of patients & 1040 & 14 & & & \\
\hline $\begin{array}{l}\text { Osteopenia } \\
\text { Preop bisphosphonate }\end{array}$ & & & & & \\
\hline No & 311 & 7 & $2.44(0.63-4.22)$ & Ref & \\
\hline Yes & 97 & 0 & $0.00(0.00-0.00)$ & - & \\
\hline Osteoporosis & & & & & \\
\hline Preop bisphosphonate & & & & & \\
\hline No & 262 & 4 & $1.89(0.03-3.71)$ & Ref & \\
\hline Yes & 370 & 3 & $0.85(0.00-1.80)$ & $0.96(0.20-4.55)$ & 0.964 \\
\hline
\end{tabular}

${ }^{*} \mathrm{CIF}$ calculated as 1 minus the Kaplan-Meier estimator.

† Cox regression model adjusted for age, BMI, sex, admitting diagnosis, operative time, surgical approach, BMP use, and number of levels fused. 
occurred with pharmacotherapy. We believe that future studies of larger cohorts of osteopenic patients may reveal meaningful information on the use of preoperative bisphosphonates.

In our osteoporotic cohort we found that the 2-year incidence of operative nonunions was $0.85 \%$ for patients with preoperative bisphosphonates and $1.89 \%$ for those without. After adjusting for covariates, including the type of surgical approach used, the number of levels fused, and the use of BMP (Table 3), no difference was observed in risk for operative nonunions. Hence, in the present study the use of preoperative bisphosphonates had no effect on the operative nonunion rates for patients with osteoporosis, and these findings join the mounting evidence that supports this conclusion. . $^{11,12,15,17,21,22}$

\section{Bisphosphonates Versus Controls-No Effect on Fusion Rates}

Two animal studies ${ }^{23,25}$ and 6 human studies ${ }^{11,12,15,17,21,22}$ have shown no effect of bisphosphonates on fusion rates. In 2012, Li et al. ${ }^{17}$ reported their work on a randomized, double-blind, placebo-controlled trial involving patients ( $\geq 50$ years of age) who underwent TLIFs. Eighty-two patients were randomly divided to receive ZA or placebo 3 days after their surgery. $\mathrm{Li}$ et al. found an $11.5 \%$ nonunion rate in the ZA group compared with a $14.5 \%$ rate in the placebo group at 12 months postoperatively, a difference that was not statistically significant. Similar results were seen by Chen et al. ${ }^{11}$ in 2016 in their RCT. Seventy-nine osteoporotic patients received either ZA or saline infusion (controls) after PLIFs. After 1 year, $82 \%$ of patients in the ZA group and $83 \%$ in the control group had solid fusion, with the difference showing no statistical significance.

There have been 4 comparative studies. ${ }^{12,15,21,22}$ Park et al., ${ }^{22}$ in 2013, did a retrospective review of patients with 1- or 2-level PLFs who received ZA or a placebo. Ohtori et al. ${ }^{21}$ explored the efficacy of teriparatide versus risedronate to reduce the incidence of pedicle screw loosening in PLF patients. Kim et al. ${ }^{15}$ studied 1-level PLIFs with a mean follow-up of 33.8 months in patients who received alendronate or a placebo. Ding et al. ${ }^{12}$ also studied the effects of ZA in treated patients versus a control group. All studies showed no statistically significant difference in radiographic fusion rates between patients with and controls without bisphosphonate use.

\section{Comparison Between Our Study and Previous Studies}

All of the studies previously presented so far support our conclusions, but there are some similarities and differences between our study and previous studies. Similarities include the finding that fusion types in our study patients were not a factor that influenced the effect of bisphosphates on nonunion rates. This finding is supported by several studies that used different types of fusions, 1 study with PLIFs, ${ }^{11} 2$ with TLIFs, ${ }^{12,17}$ and 3 with PLFs. ${ }^{15,21,22}$ The other similarity was that our study included various bisphosphonates, similarly to several studies that each used different bisphosphonates and for which the results led to similar conclusions: ZA was used in 3 studies s $^{11,12,17,22}$ and alendronate $^{12}$ and risedronate ${ }^{21}$ were used in 1 study each.

The differences between our study and other studies include the follow-up time, which in our study averaged 5.2 years, with a maximum follow-up of 9.2 years, much longer than any of the previous studies. Park et al..$^{22}$ had the shortest follow-up time, 6 months; 3 studies had 12-month follow-ups,,$^{11,17,21}$ and the rest had $\geq 2$ years of follow-up..$^{12,15}$ Our study also had the largest cohort of osteoporotic patients (632 patients), with 82 patients being the highest number among the other studies ${ }^{17}$ and 44 patients $^{15}$ the lowest.

There are, however, 2 significant differences between our studies and other studies. The first was the variation in the timing of when bisphosphonates were given. ZA was given 3 days postsurgery in 3 studies, ,11,12,17 2 weeks postoperatively in 1 study, ${ }^{22}$ and in a study by Ohtori et al., ${ }^{21}$ risedronate was given once a day from 2 months before surgery until 10 months after surgery. In our study bisphosphonates were only given preoperatively. We think that further studies should be done to determine if various preoperative and postoperative timings of bisphosphonate administration affect the nonunion rates.

Another significant difference was the method used to measure nonunions. All of the previous studies used only radiographic nonunions as their outcome measures. We know that the correlation between radiographic fusion masses and in vivo fusions has not been resolved, with studies showing that the quality assessment of the fusion mass may not be very rigorous when evaluated by manual palpation. ${ }^{13,16,19}$ Blumenthal and Gill ${ }^{26}$ reported agreement between these methods to be $69 \%$ after surgery; the falsepositive rate was $42 \%$ and the false-negative rate was $29 \%$. Kant et al. ${ }^{42}$ reported similar findings in 75 patients undergoing spinal instrumentation removal and quoted a positive correlation between radiographs and surgical findings of only $68 \%$.

In our study we used reoperation for symptomatic nonunions (operative nonunions), which addresses some of the discrepancies between radiographic and in vivo nonunion assessments by combing the radiographic findings with clinical data. However, there may have been cases in which patients had in vivo nonunion (fibrous nonunion) but were clinically asymptomatic or had no symptoms and decided not to have surgery or were not offered surgery. In general, due to the overestimation of radiographic nonunions, operative nonunion rates are lower, as shown in this study $(0 \%-2.244 \%)$ as well as other studies. ${ }^{30-33} \mathrm{We}$ believe, however, that operative nonunion rates combine clinical and radiographic data and provide a more meaningful outcome measure that is clinically relevant to the patients, surgeons, and healthcare payers and for examining the cost-effectiveness of different fusion choices.

\section{Bisphosphonates Versus Controls-Decrease in Fusion Rates}

There have been 3 animal studies and no human studies that have shown that bisphosphonates have a negative effect on fusion rates. Lehman et al. ${ }^{16}$ showed in a rabbit model that alendronate sodium inhibits or delays bone fusion after PLFs. In a rat model, Huang et al. ${ }^{14}$ found that with alendronate treatment, although the masses appeared radiographically larger and denser, with manual palpation the quality of the fusion was lower in the alendronate- 
treated group compared with the control group. Similarly, Babat et al. ${ }^{8}$ compared pamidronate (bisphosphonate) to calcitonin treatment in New Zealand white rabbits who had PLFs and found that the pamidronate group had mechanically less-robust fusion masses.

\section{Bisphosphonates Versus Controls-Increase in Fusion Rates}

There have been 2 animal $^{9,20}$ and 3 human studies ${ }^{18,19,24}$ that showed the beneficial effects of bisphosphonates for the promotion of lumbar spinal fusions. In humans, Nagahama et al ${ }^{19}$ presented the first RCT comparing alendronate-treated patients with controls in 44 osteoporotic patients undergoing 1-level PLIFs. The authors found bridging bone and solid fusions in $95 \%$ of the patients in the alendronate group but in only $65 \%$ of the control group. Tu et al. ${ }^{24}$ in 2014, retrospectively analyzed 64 osteoporotic patients (32 with intravenous ZA infusion and 32 controls) who underwent lumbar interbody fusion surgery for lumbar degeneration. Solid fusions were found in $75 \%$ of the ZA group but in only $56 \%$ of the control group after a 2-year follow-up. Liu et al., ${ }^{18}$ in 2018, in their meta-analysis paper of $3 \mathrm{RCTs}^{11,17,19}$ and 4 comparative studies, ${ }^{12,21,22,24}$ used multiple outcome measures to evaluate bisphosphonate use in osteoporotic patients. They found higher radiographic fusion rates in the bisphosphonate group compared with the control group. In addition, pedicle screw loosening was significantly lower in the bisphosphonate group compared with the control group, and the pooled incidence of cage subsidence in the bisphosphonate group was significantly lower than that in the pooled control group.

\section{Strengths and Limitations of Our Study}

We acknowledge limitations in our study. First, as in many studies using registry data, the lack of randomization could have introduced different kinds of biases. While we attempted to control for a number of covariates through our multivariable analysis, residual confounding due to unmeasured factors may be possible. Selection bias for the type of treatment chosen by the surgeon may contribute to this, but with 93 surgeons (neurosurgeons and orthopedic spine surgeons) providing patients to the registry, their indications are likely representative of spine surgeons in the general community. Using data from the Swedish Spine Register, Endler et al. ${ }^{43}$ also noted that using registry data increases the generalizability of the results because they represent those of the routine surgeries performed.

Second, although the registry database relies on accurate entry by our surgeons of data for diagnosis, surgical procedures, and complications, in our study, we had to rely on coding data (ICD or CPT) for the diagnosis of osteoporosis or osteopenia. We believe the data are accurate based on sampling of the database for correlations between DEXA scan data and diagnosis, as well as the requirement for accurate coding for billing within our healthcare system, since these areas are subject to internal and external audits.

Another limitation is that individual bisphosphonates could not be evaluated, nor could the preoperative timing, duration, or dosage of the medication. Further, inferences cannot be made regarding starting bisphosphonates postoperatively and risk for operative nonunion, as this was not evaluated. However, our definition of preoperative bisphosphonate use is consistent with that used in previously published studies. ${ }^{44}$

There was variability with regard to the different types of bone grafts used (allograft versus autograft or both). Since rates of operative nonunion events were so low in both the bisphosphonate users and nonusers, we do not think these factors significantly affected the outcome, and most likely they were equally distributed between the groups. This is evident in our BMP use, which we measured and found to be equally distributed in both groups (Tables 1 and 2).

Our study strengths include one of the largest cohorts of patients with the longest follow-up periods in the literature comparing the effects of preoperative bisphosphonates on osteopenia and osteoporotic patients. This report also introduces for the first time, to our knowledge, the use of operative nonunion rates to evaluate the effects of bisphosphonates. We believe this method is an important alternative to the use of radiographic nonunions to define fusion rates, a method that has limitations, as we noted previously.

Finally, our study has the advantage of including patients from the spine registry of an integrated healthcare system with no dependence on industry support, and features varied patient demographics with surgical indications. It presents a more realistic and unbiased assessment of routine lumbar spine fusion care and represents a heterogeneous population with varied indications and surgical techniques.

\section{Conclusions}

To our knowledge, this study presents one of the largest series of patients with the diagnoses of osteopenia and osteoporosis in whom the effects of preoperative bisphosphonate use on lumbar fusions were evaluated using operative nonunion rates as an outcome measure. We found that preoperative bisphosphonate use had no effect on the operative nonunion rates for patients with osteoporosis. We were unable to make similar conclusions for the osteopenia patients because of the low nonunion frequency. We believe further studies are warranted to determine if preoperative and postoperative timing of bisphosphonate use in osteoporotic or osteopenic patients has any effect on lumbar fusion rates.

\section{References}

1. Chin DK, Park JY, Yoon YS, et al. Prevalence of osteoporosis in patients requiring spine surgery: incidence and significance of osteoporosis in spine disease. Osteoporos Int. 2007; 18(9):1219-1224.

2. Etebar S, Cahill DW. Risk factors for adjacent-segment failure following lumbar fixation with rigid instrumentation for degenerative instability. J Neurosurg. 1999;90(2)(suppl): $163-169$.

3. Halvorson TL, Kelley LA, Thomas KA, et al. Effects of bone mineral density on pedicle screw fixation. Spine (Phila Pa 1976). 1994;19(21):2415-2420.

4. Okuda S, Oda T, Miyauchi A, et al. Surgical outcomes of 
posterior lumbar interbody fusion in elderly patients. $J$ Bone Joint Surg Am. 2006;88(12):2714-2720.

5. Cotts KG, Cifu AS. Treatment of osteoporosis. JAMA. 2018; 319(10):1040-1041.

6. Russell RG, Watts NB, Ebetino FH, Rogers MJ. Mechanisms of action of bisphosphonates: similarities and differences and their potential influence on clinical efficacy. Osteoporos Int. 2008;19(6):733-759.

7. Schimmer RC, Bauss F. Effect of daily and intermittent use of ibandronate on bone mass and bone turnover in postmenopausal osteoporosis: a review of three phase II studies. Clin Ther. 2003;25(1):19-34

8. Babat LB, McLain R, Milks R, et al. The effects of the antiresorptive agents calcitonin and pamidronate on spine fusion in a rabbit model. Spine J. 2005;5(5):542-547.

9. Bransford R, Goergens E, Briody J, et al. Effect of zoledronic acid in an L6-L7 rabbit spine fusion model. Eur Spine J. 2007;16(4):557-562.

10. Buerba RA, Sharma A, Ziino C, et al. Bisphosphonate and teriparatide use in thoracolumbar spinal fusion: a systematic review and meta-analysis of comparative studies. Spine (Phila Pa 1976). 2018;43(17):E1014-E1023.

11. Chen F, Dai Z, Kang Y, et al. Effects of zoledronic acid on bone fusion in osteoporotic patients after lumbar fusion. Osteoporos Int. 2016;27(4):1469-1476.

12. Ding Q, Chen J, Fan J, et al. Effect of zoledronic acid on lumbar spinal fusion in osteoporotic patients. Eur Spine J. 2017; 26(11):2969-2977.

13. Hirsch BP, Unnanuntana A, Cunningham ME, Lane JM. The effect of therapies for osteoporosis on spine fusion: a systematic review. Spine J. 2013;13(2):190-199.

14. Huang RC, Khan SN, Sandhu HS, et al. Alendronate inhibits spine fusion in a rat model. Spine (Phila Pa 1976). 2005; 30(22):2516-2522.

15. Kim SM, Rhee W, Ha S, et al. Influence of alendronate and endplate degeneration to single level posterior lumbar spinal interbody fusion. Korean J Spine. 2014;11(4):221-226.

16. Lehman RA Jr, Kuklo TR, Freedman BA, et al. The effect of alendronate sodium on spinal fusion: a rabbit model. Spine $J$. 2004;4(1):36-43.

17. Li C, Wang HR, Li XL, et al. The relation between zoledronic acid infusion and interbody fusion in patients undergoing transforaminal lumbar interbody fusion surgery. Acta Neurochir(Wien). 2012;154(4):731-738.

18. Liu WB, Zhao WT, Shen P, Zhang FJ. The effects of bisphosphonates on osteoporotic patients after lumbar fusion: a meta-analysis. Drug Des Devel Ther. 2018;12:2233-2240.

19. Nagahama K, Kanayama M, Togawa D, et al. Does alendronate disturb the healing process of posterior lumbar interbody fusion? A prospective randomized trial. J Neurosurg Spine. 2011;14(4):500-507.

20. Nakao S, Minamide A, Kawakami M, et al. The influence of alendronate on spine fusion in an osteoporotic animal model. Spine (Phila Pa 1976). 2011;36(18):1446-1452.

21. Ohtori S, Inoue G, Orita S, et al. Comparison of teriparatide and bisphosphonate treatment to reduce pedicle screw loosening after lumbar spinal fusion surgery in postmenopausal women with osteoporosis from a bone quality perspective. Spine (Phila Pa 1976). 2013;38(8):E487-E492.

22. Park YS, Kim HS, Baek SW, et al. The effect of zoledronic acid on the volume of the fusion-mass in lumbar spinal fusion. Clin Orthop Surg. 2013;5(4):292-297.

23. Takahata M, Ito M, Abe Y, et al. The effect of anti-resorptive therapies on bone graft healing in an ovariectomized rat spinal arthrodesis model. Bone. 2008;43(6):1057-1066.

24. Tu CW, Huang KF, Hsu HT, et al. Zoledronic acid infusion for lumbar interbody fusion in osteoporosis. J Surg Res. 2014; 192(1):112-116.

25. Xue Q, Li H, Zou X, et al. The influence of alendronate treat- ment and bone graft volume on posterior lateral spine fusion in a porcine model. Spine (Phila Pa 1976). 2005;30(10): $1116-1121$

26. Blumenthal SL, Gill K. Can lumbar spine radiographs accurately determine fusion in postoperative patients? Correlation of routine radiographs with a second surgical look at lumbar fusions. Spine (Phila Pa 1976). 1993;18(9):1186-1189.

27. Brodsky AE, Kovalsky ES, Khalil MA. Correlation of radiologic assessment of lumbar spine fusions with surgical exploration. Spine (Phila Pa 1976). 1991;16(6)(suppl):S261-S265.

28. Dawson E, Bae HW, Burkus JK, et al. Recombinant human bone morphogenetic protein-2 on an absorbable collagen sponge with an osteoconductive bulking agent in posterolateral arthrodesis with instrumentation. A prospective randomized trial. J Bone Joint Surg Am. 2009;91(7):1604-1613.

29. Singh K, Phillips FM, Park DK, et al. Factors affecting reoperations after anterior cervical discectomy and fusion within and outside of a Federal Drug Administration investigational device exemption cervical disc replacement trial. Spine J. 2012;12(5):372-378.

30. Guppy KH, Paxton EW, Harris J, et al. Does bone morphogenetic protein change the operative nonunion rates in spine fusions? Spine (Phila Pa 1976). 2014;39(22):1831-1839.

31. Guppy KH, Harris J, Paxton LW, et al. Reoperation rates for symptomatic nonunions in anterior cervical fusions from a national spine registry. Spine (Phila Pa 1976). 2015;40(20): 1632-1637.

32. Guppy KH, Harris J, Chen J, et al. Reoperation rates for symptomatic nonunions in posterior cervical (subaxial) fusions with and without bone morphogenetic protein in a cohort of 1158 patients. J Neurosurg Spine. 2016;24(4): $556-564$

33. Guppy KH, Harris J, Chen J, et al. Reoperation rates for symptomatic nonunions in posterior cervicothoracic fusions with and without bone morphogenetic protein in a cohort of 450 patients. J Neurosurg Spine. 2016;25(3):309-317.

34. Karter AJ, Ferrara A, Liu JY, et al. Ethnic disparities in diabetic complications in an insured population. JAMA. 2002; 287(19):2519-2527.

35. Koebnick C, Langer-Gould AM, Gould MK, et al. Sociodemographic characteristics of members of a large, integrated health care system: comparison with US Census Bureau data. Perm J. 2012;16(3):37-41.

36. Paxton EW, Inacio MC, Kiley ML. The Kaiser Permanente implant registries: effect on patient safety, quality improvement, cost effectiveness, and research opportunities. Perm J. 2012;16(2):36-44.

37. Boonen S, Vanderschueren D, Venken K, et al. Recent developments in the management of postmenopausal osteoporosis with bisphosphonates: enhanced efficacy by enhanced compliance. J Intern Med. 2008;264(4):315-332.

38. Iwamoto J, Takeda T, Sato Y. Effects of antifracture drugs in postmenopausal, male and glucocorticoid-induced osteoporosis-usefulness of alendronate and risedronate. Expert Opin Pharmacother. 2007;8(16):2743-2756.

39. Orwoll ES, Miller PD, Adachi JD, et al. Efficacy and safety of a once-yearly i.v. infusion of zoledronic acid $5 \mathrm{mg}$ versus a once-weekly 70-mg oral alendronate in the treatment of male osteoporosis: a randomized, multicenter, double-blind, active-controlled study. J Bone Miner Res. 2010;25(10): 2239-2250.

40. Reid IR, Horne AM, Mihov B, et al. fracture prevention with zoledronate in older women with osteopenia. $N$ Engl J Med. 2018;379(25):2407-2416.

41. Iqbal SM, Qamar I, Zhi C, et al. Role of bisphosphonate therapy in patients with osteopenia: a systemic review. Cureus. 2019;11(2):e4146.

42. Kant AP, Daum WJ, Dean SM, Uchida T. Evaluation of lumbar spine fusion. Plain radiographs versus direct surgical 
exploration and observation. Spine (Phila Pa 1976). 1995; 20(21):2313-2317.

43. Endler P, Ekman P, Möller H, Gerdhem P. Outcomes of posterolateral fusion with and without instrumentation and of interbody fusion for isthmic spondylolisthesis: a prospective study. J Bone Joint Surg Am. 2017;99(9):743-752.

44. Prieto-Alhambra D, Javaid MK, Judge A, et al. Association between bisphosphonate use and implant survival after primary total arthroplasty of the knee or hip: population based retrospective cohort study. BMJ. 2011;343:d7222.

\section{Disclosures}

The authors report no conflict of interest concerning the materials or methods used in this study or the findings specified in this paper.

\section{Author Contributions}

Conception and design: Guppy. Acquisition of data: Guppy, Chan, Prentice, Harris. Analysis and interpretation of data:
Guppy, Chan, Prentice, Harris, Brara. Drafting the article: Guppy, Norheim, Harris, Brara. Critically revising the article: Guppy, Prentice, Norheim, Brara. Reviewed submitted version of manuscript: Guppy, Chan, Prentice, Norheim, Brara. Approved the final version of the manuscript on behalf of all authors: Guppy. Statistical analysis: Chan, Prentice, Harris. Administrative/technical/material support: Prentice, Harris, Brara. Study supervision: Guppy, Brara.

\section{Correspondence}

Kern H. Guppy: The Permanente Medical Group, Sacramento, CA.kguppy@yahoo.com. 